\title{
ON THE MEAN CONVERGENCE OF MARKOV OPERATORS
}

\author{
by ROBERT E. ATALLA
}

(Received 21st July 1973)

\section{Introduction}

Throughout the paper, $T$ will be a Markov operator on $C(X)\left(X\right.$ compact $\left.T_{2}\right)$, i.e. a continuous positive operator such that $T e=e$ ( $e$ the unit function). $P$ will be the set of Borel probability measures on $X$, which we shall often think of as linear functionals on $C(X)$, and $P_{T}=\left\{m \in P: T^{\prime} m=m\right\}$, where $T^{\prime}$ is the adjoint of $T$. Let

$$
F=\text { closure } \cup\left\{\text { carrier }(m): m \in P_{T}\right\} \text {. }
$$

Our main result, Theorem 3.1, is the following. Suppose $S$ is a Markov projection on $C(X)$ such that $T_{n} f \rightarrow 0$ iff $S f=0$, where $T_{n}=(1 / n)\left(T+\ldots+T^{n}\right)$. Then $T$ and $S$ induce operators $T_{0}$ and $S_{0}$ on $C(F)$ such that $\left(T_{0}\right)_{n} f \rightarrow S_{0} f$ for all $f \in C(F)$.

Our results may be motivated as follows. If $T$ is a continuous operator on a topological vector space and $S$ is a projection, it is an easy exercise to prove that for $T_{n} x \rightarrow S x$ (all $x$ ) to occur, it is necessary and sufficient that

(i) $T_{n} x \rightarrow 0$ iff $S x=0$, and

(ii) $S T=T S=S$.

Now (i) easily implies $S T=S$. (We must show that if (i) holds, then $0=S(T-\mathrm{I})$, or $T x-x \in \operatorname{kernel}(S)$ for all $x$. But $T_{n}(T x-x) \rightarrow 0$, so

$$
S(T x-x)=0 \text {, }
$$

by condition (i).) Thus (ii) may be replaced by (ii)' $T S=S$. What our main result shows is that in the special case of Markov operators on $C(X)$, (ii) may be discarded altogether, and positive convergence results still obtained.

Section 4 will show how condition (i) arises naturally in the theory of regular matrices.

We shall assume throughout the paper that $S$ and $T$ are Markov operators such that

$$
\text { (I) } T_{n} f \rightarrow 0 \text { iff } S f=0 \quad(f \in C(X)) \text {. }
$$

\section{Preliminaries}

This section is devoted to lemmas needed for the main result in Section 3. The following notation will be used. If $x \in X$, let $t_{x}$ be the element of $P$ representing the functional $f \rightarrow T f(x)(f \in C(X))$. Thus, $T f(x)=\int f d t_{x}$. Likewise, $S f(x)=\int f d s_{x}:$ 'Recall that $P_{T}=\left\{m \in P: T^{\prime} m=m\right\}$. 
We shall assume throughout this section that $S$ and $T$ are operators satisfying condition (I) of the Introduction.

Remark 2.1. We proved in the Introduction that condition (I) implies that $S T=S$, so that for each $x \in X$ and $f \in C(X)$ we have $\int f d s_{x}=\int T f d s_{x}$. Thus, $S^{\prime} \delta_{x}=S_{x} \in P_{T}$ (where $\delta_{x}$ is the point mass at $x$ ), and hence $S^{\prime} P \subset P_{T}$.

Our first result shows that if $S^{2}=S$, then $S^{\prime} P=P_{T}$. The condition $S^{2}=S$ is the hypothesis for all results after the first, although it may be of interest to note that the results are valid under the weaker assumption that $S^{\prime} P=P_{T}$.

Lemma 2.2. (a) $S^{2}=S$ iff $P_{T}=P_{S}$.

(b) Hence if $S^{2}=S$, then $S^{\prime} P=P_{T}$.

Proof. (a) First we note that in any case, $P_{S} \subset P_{T}$. To show this, recall that $S T=S$ (Remark 2.1). If $m \in P_{S}$, then $T^{\prime} m=T^{\prime} S^{\prime} m=S^{\prime} m=m$, i.e. $m \in P_{T}$.

Assume $S^{2}=S$. If $f \in C(X)$, then $S f-f \in \operatorname{kernel}(S)$, so by condition (I) we have $T_{n}(S f-f) \rightarrow 0$. If $m \in P_{T}$, then $m(S f-f)=m\left(T_{n}(S f-f)\right) \rightarrow 0$, so $m(S f)=m(f)$, i.e. $m \in P_{S}$. Thus, $P_{T}=P_{S}$.

Assume $P_{T}=P_{S}$. Let $x \in X$. Since $S T=S$, we have $\int T f d s_{x}=\int f d s_{x}$ for all $f \in C(X)$, and hence $s_{x} \in P_{T}=P_{S}$, whence $S^{\prime} s_{x}=s_{x}$. This implies $S^{2}=S$.

(b) Assume $S^{2}=S$. We know that $S^{\prime} P \subset P_{T}$. If $m \in P_{T}$, then by $(a), S^{\prime} m=m$, so $m \in S^{\prime} P$. Thus $S^{\prime} P=P_{T}$.

Lemma 2.3. Assume $S^{2}=S$. Then (a) all extreme points of $P_{T}$ have the form $s_{x}$ for some $x \in X$, and $(b)$

$$
F=\operatorname{cl} \cup\left\{\operatorname{car}\left(s_{x}\right): s_{x} \in\left(P_{T}\right)^{e}\right\},
$$

where $\left(P_{T}\right)^{e}$ is the set of extreme points of $P_{T}$.

Proof. (a) If $m \in\left(P_{T}\right)^{e}$, then since $S^{\prime} P=P_{T}$ (by Lemma $2.2(b)$ ), the set $\left(S^{\prime}\right)^{-1}(m)=\left\{\rho \in P: S^{\prime} \rho=m\right\}$ is a non-empty face of $P$, and hence contains an extreme point (by, e.g., Theorem 9 on page 9 of (3)). Since all extreme points of $P$ have the form $\delta_{x}(x \in X)$, there exists $x \in X$ with $m=S^{\prime} \delta_{x}=s_{x}$.

(b) Let $F_{0}$ be the set in question. Clearly, $F_{0} \subset F$. Suppose $y \in F \backslash F_{0}$. Then there exists a non-negative $f \in C(X)$ with $f=0$ on $F_{0}$ and $f(y)>0$. Then $m(f)>0$ for some $m \in P_{T}$, while $(a)$ implies $\rho(f)=0$ for each $\rho \in\left(P_{T}\right)^{e}$. But this is impossible, by the Krein-Milman theorem. Hence $F_{0}=F$.

Lemma 2.4. Assume $S^{2}=S$. Let $F_{T}(F)=\{f \in C(X): T f|F=f| F\}$. Then $S(C(X)) \subset F_{T}(F)$.

Proof. Since $S^{2}=S$, it follows that $s_{x} \in P_{S}$ for each $x \in X$. Furthermore, Lemma 2.2 implies $P_{T}=P_{S}$, and Lemma $2.3(a)$ implies that each extreme point of $P_{S}$ has the form $s_{x}$ for some $x \in X$. Let $s_{x}$ be a fixed extreme point of $P_{S}$. By Theorem 1.11 of (4), $\operatorname{car}\left(s_{x}\right)$ is contained in an " $S$-ergodic set", i.e. a set of 
constancy for any $g \in C(X)$ such that $S g=g$. Since $S^{2}=S$, it follows that $S f$ is constant (say $t$ ) on $\operatorname{car}\left(s_{x}\right)$ for any $f \in C(X)$. If $\mathrm{y} \in \operatorname{car}\left(s_{x}\right)$, then since $s_{x} \in P_{T}$ we have car $\left(t_{y}\right) \subset \operatorname{car}\left(s_{x}\right)(4$, Theorem 1.3), and hence

$$
T(S f)(y)=t=S f(y) \text {. }
$$

Lemma $2.3(b)$ implies $T(S f)|F=S f| F$. Hence $S f \in F_{T}(F)$ for each $f \in C(X)$.

Remark 2.5. The preceding proof suggests the following: let $S$ be a Markov projection on $C(X)$. If $S f=0$, then $S(f S g)=0$ for all $g \in C(X)$.

Proof. Let $s_{x}$ be an extreme point of $P_{S}=S^{\prime} P$. Then for each $g \in C(X)$, $S g$ is constant (say $t)$ on car $\left(s_{x}\right)$, and hence

$$
S(f S g)(x)=\int f(w) S g(w) d s_{x}(w)=t \int f(w) d s_{x}(w)=0 .
$$

By Krein-Milman, $S(f S g)(y)=\int f(w) S g(w) d s_{y}(w)=0$ for all $\mathrm{y} \in X$, since each $s_{y} \in P_{S}$.

Lemma 2.6. Assume $S^{2}=S$. If $f=0$ on $F$, then $T f=0$ on $F$, and $S f=0$ everywhere.

Proof. To show $T f=0$ on $F$ we note that since $s_{x} \in P_{T}$ for each $x$, $\operatorname{car}\left(s_{x}\right)$ is $T$-self-supporting (4, Theorem 1.3), and hence, by (4, Theorem 1.1), if $g=0$ on car $\left(s_{x}\right)$, then $T g=0$ on $\operatorname{car}\left(s_{x}\right)$. Thus, since $f=0$ on $F$ we have $T f=0$ on each $\operatorname{car}\left(s_{x}\right)$, and hence $T f=0$ on $F$, by Lemma $2.3(b)$.

To show $S f(x)=0$ for all $x$, it is enough to show car $\left(s_{x}\right) \subset F$ for each $x \in X$. By Remark 2.1, $s_{x} \in P_{T}$ for each $x \in X$, and hence $\operatorname{car}\left(s_{x}\right) \subset F$ by the definition of $F$.

\section{Mean convergence theorem}

In this section we assume condition (I), and also that $S^{2}=S$. By Lemma 2.6, $f \mid F=0$ implies $T f \mid F=0$ and $S f \mid F=0$. We define $T_{0}$ on $C(F)$ by $T_{0} f(x)=T f(x)$ for each $f \in C(F)$ and $x \in F$, where $f \in C(X)$ is any extension of $f$. Likewise, $S_{0} f=S \vec{f}$.

Since $f \mid F=0$ implies $T f|F=S f| F=0$, the set $F$ is both $T$-selfsupporting and $S$-self-supporting, i.e. $x \in F$ implies car $\left(t_{x}\right) \subset F$ and $\operatorname{car}\left(s_{x}\right) \subset F$ (4, Theorem 1.1). Hence for $f \in C(F)$ and $x \in F$ we have

$$
T_{0} f(x)=T f(x)=\int f d t_{x}=\int f d t_{x}, \quad \text { and } S_{0} f=\int f d s_{x} .
$$

Theorem 3.1. Assume (I) $S f=0$ iff $T_{n} f \rightarrow 0$, and (II) $S^{2}=S$. Then $\left(T_{0}\right)_{n} f \rightarrow S_{0} f$ for each $f \in C(F)$.

Proof. We first prove the following:

(i) $T_{0} S_{0}=S_{0}$,

(ii) $S_{0}^{2}=S_{0}$, and

(iii) $S_{0} f=0$ implies $\left(T_{0}\right)_{n} f \rightarrow 0$. 
For (i), if $f \in C(F)$, then by Lemma 2.4, $S \bar{f} \in F_{T}(F)$, and hence for each $y \in F, T(S f)(y)=S \tilde{f}(y)$, or $T_{0}\left(S_{0} f\right)(y)=S_{0} f(y)$.

For (ii), if $f \in C(F), S_{0}{ }^{2} f=S^{2} \bar{f}|F=S f| F=S_{0} f$.

For (iii), let $x \in X$. Then since $S f=0$ on $\operatorname{car}\left(s_{x}\right)(\subset F)$, we have

$$
S \vec{f}(x)=S^{2} \vec{f}(x)=\int S f d s_{x}=0 .
$$

By condition (I), $\left(T_{0}\right)_{n} f=T_{n} \bar{f} \mid F \rightarrow 0$.

We now show that if $f \in C(F)$, then $\left(T_{0}\right)_{n} f \rightarrow S_{0} f$. Since $S_{0}{ }^{2}=S_{0}$, we have $S_{0} f-f \in \operatorname{kernel}\left(S_{0}\right)$, and hence (iii) implies $\left(T_{0}\right)_{n}\left(S_{0} f-f\right) \rightarrow 0$. Using (i), we have $\left(T_{0}\right)_{n} f-S_{0} f=\left(T_{0}\right)_{n}\left(f-S_{0} f\right) \rightarrow 0$.

Theorem 3.2. Suppose the conditions of Theorem 3.1 are satisfied, and that $T$ also satisfies the condition

$$
\operatorname{car}\left(t_{x}\right) \subset F \text { for all } x \in X .
$$

Then $T_{n} f \rightarrow T S f$ for all $f \in C(X)$ (and hence TS is a projection).

Proof. By Theorem 3.1 we have $T_{n} f|F \rightarrow S f| F$ for each $f \in C(X)$. Let $R_{n}=(1 / n)\left(I+T+\ldots+T^{n-1}\right)$, so $T_{n}=T R_{n}$. Clearly, $R_{n} f|F \rightarrow S f| F$ for each $f \in C(X)$. If $x \in X$,

$$
\begin{aligned}
T_{n} f(x)=T\left(R_{n} f\right)(x) & =\int_{F} R_{n} f d t_{x} \\
& \rightarrow \int_{F} S f d t_{x}=T(S f)(x),
\end{aligned}
$$

by dominated convergence. By Theorem 1.1 of (2), $T_{n} f \rightarrow T(S f)$ uniformly.

\section{Final remarks}

We conclude with a remark on how condition (I) arises naturally in matrix summability. Let $T=\left(t_{m n}\right)$ be a regular matrix, considered as a linear operator on $C^{*}(N)$, and $m_{T}$ the set of $T$-invariant means on $C^{*}(N)$, as defined in (1). $f \in C^{*}(N)$ is T-almost convergent if $\rho_{1}(f)=\rho_{2}(f)$ for all $\rho_{1}, \rho_{2} \in m_{T}$. Let $V_{T}$ be the space of $T$-almost convergent functions. By a well-known result of G. G. Lorentz, if $T$ is the shift matrix $(T f(n)=f(n+1))$, then $V_{T}$ is not equal to the bounded convergence field $C_{S}$ of any regular matrix $S$. It is natural to ask under what circumstances the equation $V_{T}=C_{S}$ is possible. (This question has been asked by J. P. Duran. To the author's knowledge it remains open.)

As in (1), let $T_{1}$ and $S_{1}$ be the operators induced on $C(\beta N \backslash N)$ by the matrices $T$ and $S$. Then it can be shown that the condition $V_{T}=C_{S}$ is equivalent to (I) $\left(T_{1}\right)_{n} f \rightarrow 0$ iff $S_{1} f=0(f \in C(\beta N \backslash N))$. (Necessity is easy, and sufficiency follows from an easy generalisation of the Mazur-Orlicz consistency theorem.) 


\section{REFERENCES}

(1) R. Atalla, On the inclusion of a bounded convergence field in the space of almost convergent sequences, Glasgow Math. J. 13 (1972), 82-90.

(2) B. JAMISON, Ergodic decompositions induced by certain Markov operators, Trans. Amer. Math. Soc. 117 (1965), 451-468.

(3) G. LelBowitz, Lectures on complex function algebras (Scott, Foresman and Company, Glenview, Illinois, 1970).

(4) R. Sine, Geometric theory of a single Markov operator, Pacific J. Math 27 (1968), 155-166.

OHIO UNIVERSITY

ATHENS, OHIO 45701 